\title{
Los Estudios Generales como pedagogía multidisciplinaria en vías a la interdisciplina
}

\section{General Studies as a Multidisciplinary Teaching Becoming an Interdisciplinary Approach}

Miguel Baraona Cockerell Universidad Nacional, Sede Central Heredia, Costa Rica miguel.baraona.cockerell@una.cr

Enrique Mata Rivera Universidad Nacional, Sede Central

Heredia, Costa Rica enrique.mata.rivera@una.cr

\section{Resumen}

Durante cuatro décadas, el Centro de Estudios Generales de la Universidad Nacional (CEG-UNA) de Costa Rica ha sido cada vez más una facultad multidisciplinaria. Partió de la enseñanza convencional de las humanidades en los setenta, pero hoy abarca disciplinas en todos los campos fundamentales del conocimiento humano: ciencias sociales, ciencias naturales, filosofía, y letras y arte. Esta expansión constante de la malla curricular del CEG-UNA, mediante una oferta actual de más de 100 cursos de muy diferente índole y orientación disciplinaria (varios de ellos interdisciplinarios), ha transformado esta unidad académica en un centro de enseñanza único en el contexto de la UNA. En la actualidad, trabaja sobre una teoría práctica denominada Nuevo Humanismo

Latinoamericano. Así el CEG-UNA intenta hacer la difícil transición de la multidisciplina a una pedagogía interdisciplinaria integral y viable desde

el punto de vista de la docencia, la extensión, la producción y la investigación. En este 
artículo se describen y analizan los aspectos más relevantes de este esfuerzo pedagógico, académico y epistemológico.

Palabras clave: Estudios Generales; historia; evolución; multidisciplina; interdisciplina.

\begin{abstract}
During four decades the Center for General Studies (CEG-UNA) of the National University of Costa Rica, has become an increasingly multi-disciplinary school. It started with a conventional teaching of humanities in the seventies, but today it encompasses disciplines in all the fundamental fields of human knowledge: social sciences, natural sciences, philosophy, and literature and art. This constant expansion of the curricular offer by the CEG-UNA through more than 100 different courses of a very diverse disciplinary orientation (several of them with an interdisciplinary design) has transformed the center into a unique academic center within the context of UNA. Currently, it works on a theoretical and methodological perspective that we conceptualize as New Latin American Humanism. Thus, the CEG-UNA is attempting to make the complex transition from a multi-disciplinary approach feasible from the point of view of teaching, extension, production, and research. This article describes and analyzes the most relevant aspects of this pedagogical, academic, and epistemological.
\end{abstract}

Keywords: General studies; history; evolution; multi-discipline; inter-discipline.

\title{
Un poco de historia
}

El Centro de Estudios Generales (CEG) de la Universidad Nacional (UNA) nació junto con la Universidad en 1973. En sus orígenes, el CEG fue concebido como el corazón pedagógico de la Universidad y destinado a formar estudiantes en las humanidades tradicionales (filosofía, historia y literatura) y en algunos cursos adicionales en ciencias básicas. Hacia finales de la década de los setenta del siglo pasado, se ampliaron las formas sustantivas de accionar del CEG y se impulsaron, además de la pedagogía, la investigación y la extensión. Algunos de sus frutos quedaron consignados en las publicaciones del Centro como la "Revista de Nuevo Humanismo" y el desplegable "Tópicos del Humanismo", los cuales se publicaron durante muchos años (la primera de ambas revistas ha sido relanzada en el 2014).

A partir de 1989, se comenzó un experimento de interdisciplina, pero solo entre las tres disciplinas tradicionales y muy afines de las humanidades, y se establecieron puentes y diálogos entre la historia, la filosofia y la literatura. Este fue un valioso 
esfuerzo, pero limitaba la significación pedagógica y epistemológica de este intento. Desde principios de los noventa, el CEG se reinventa una vez más. Se abandona el enfoque exclusivo en las tres disciplinas tradicionales y se formaliza una malla curricular mucho más amplia, que incluye cursos en cuatro áreas principales: Ciencia y Tecnología, Filosofía y Letras, Ciencias Sociales, Arte y Sociedad. A mediados del 2015, el CEG comenzó a renovarse acorde con un nuevo proyecto que busca dejar atrás las cuatro áreas mencionadas y comenzar a desarrollar una malla curricular, así como de investigación, extensión y producción, gradualmente más interdisciplinaria. $\mathrm{Y}$ es este proceso que caracterizamos como el de transición de la multidisciplina a la interdisciplina, dentro del marco del paradigma transversal (transdisciplinario) del Nuevo Humanismo Latinoamericano, el que deseamos reseñar en las páginas que siguen (Fonseca, 2012).

\section{El Centro de Estudios Generales de la Universidad Nacional en la actualidad}

A mediados del 2015, el CEG de la UNA estuvo integrado por 37 académicos, quienes impartían cerca de 100 cursos, muchos diferentes y algunos, incluso, podríamos considerarlos interdisciplinarios, porque abarcan disciplinas afines o incluso distantes, como las Ciencias Naturales y las Ciencias Sociales. Los académicos provienen de disciplinas diferentes, agrupadas en las cuatro áreas principales mencionadas.

Además de la enseñanza, el CEG está abocado al desarrollo de 12 proyectos. Entre ellos se encuentran de proyectos de extensión en diferentes disciplinas como las Artes y la interdisciplina entre Ciencias Naturales y Ciencias Sociales y uno de investigación histórica y literaria. Estos proyectos son desarrollados por académicos que, en general, dedican tres cuartas partes de su tiempo laboral a la enseñanza y una cuarta parte a un proyecto específico, a menudo con apoyo de estudiantes que realizan una práctica útil con su participación. También se cuenta con un programa (conjunto de proyectos asociados en torno a una temática o eje central) que se aboca a la atención y educación a adultos mayores, llamado Programa Integral de Apoyo al Adulto mayor (PAIPAM) y que fue creado en el 2004. En este Programa laboran a tiempo parcial (entre un cuarto y medio tiempo) cerca de cuatro académicas (este número fluctúa dependiendo del número de proyectos asociados al PAIPAM) y algunos estudiantes.

En el primer cuatrimestre del 2016, el CEG-UNA comenzará a implementar un programa de maestría sobre "Humanismo, sociedad y ambiente" de carácter 
interdisciplinario. Lo consideramos como la antesala académica y pedagógica al programa de doctorado sobre "Humanismo, interdisciplina y complejidad".

\section{Niveles epistemológicos de integración disciplinarial}

En este trabajo utilizamos un marco conceptual simple, según el cual jerarquizamos el proceso de integración disciplinaria que parte de su nivel más básico, que es el de las disciplinas concebidas como compartimentos estancos y que, por la praxis misma de la ciencia y del conocimiento humano, en general, se eleva y pasa por la multidisciplina, la interdisciplina, hasta llegar al plano más complejo de la transdisciplina (figura 1).

A continuación se aporta una breve definición de los niveles epistemológicos superiores de integración disciplinaria como son: la multidisciplina, la interdisciplina y la transdisciplina. Partimos, además, del supuesto axiológico según el cual, a medida que se asciende a los niveles epistemológicos superiores, el pensamiento - es decir, el modelo de compresión de la realidad- se vuelve más complejo (García, 2006), lo cual significa un ascenso de los niveles más simples, abstractos y mecánicos, a niveles más complejos, concretos y dialécticos de conocimiento (Kosic, 1967). 


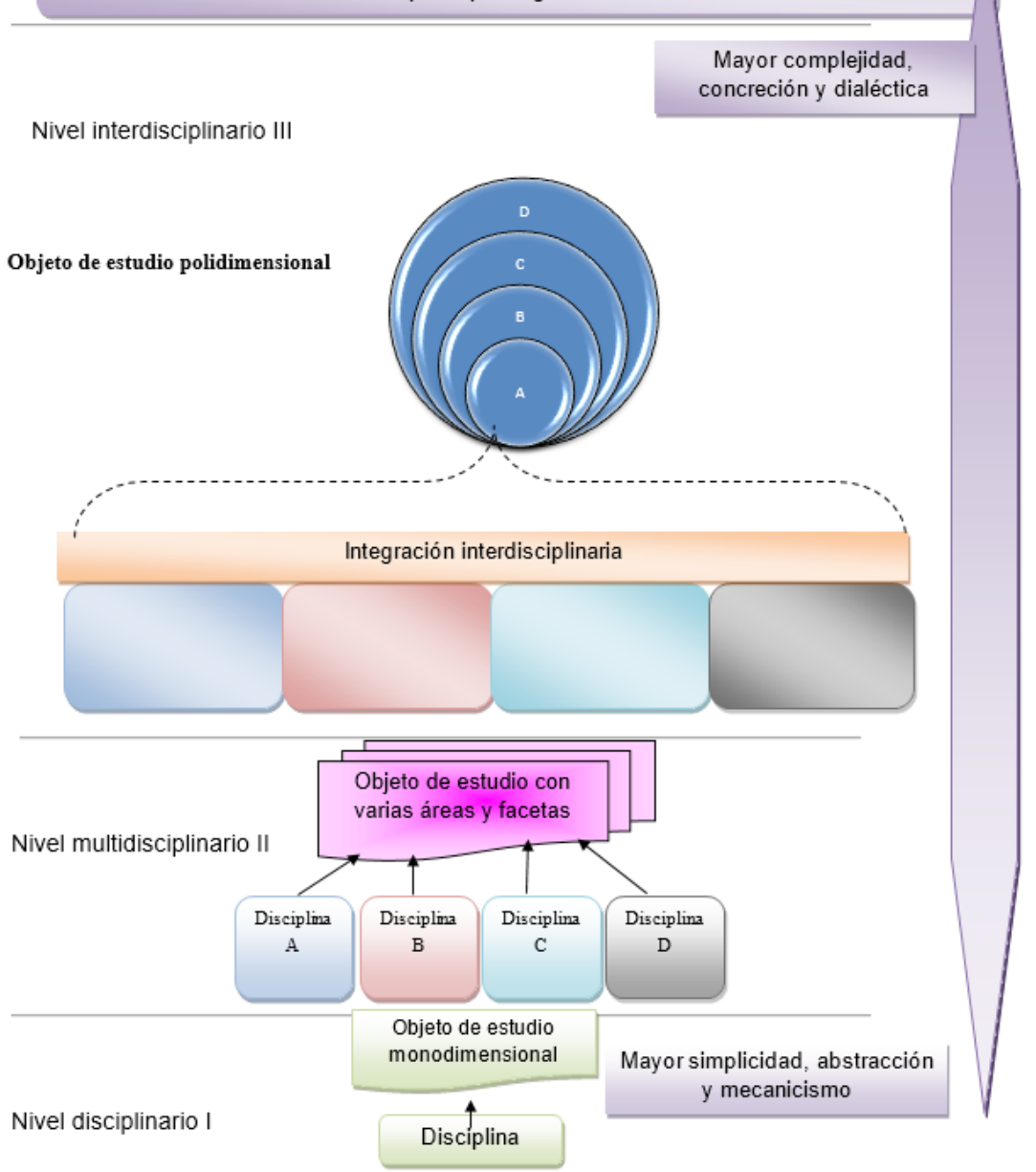

Figura 1. Niveles epistemológicos de la integración disciplinaria

\section{De la multidisciplina a la interdisciplina}

El CEG es la única unidad académica de toda la UNA que tiene un carácter ampliamente multidisciplinario y que abarca disciplinas distantes, desde el punto de vista conceptual, metodológico y en cuanto a su objeto de estudio o interés. Y siguiendo tendencias ya universales en la educación superior (Davies \& Devlin), nos proponemos, en los próximos cinco años (2015-2020), iniciar y llevar a plena 
fruición el paso de lo multidisciplinario a lo interdisciplinario, tanto en docencia, como en investigación, extensión y producción en el CEG-UNA.

Si clasificamos las distintas disciplinas del saber y la praxis humana, acorde con su afinidad, podríamos clasificarlas por la proximidad de sus objetos de estudio y sus métodos como disciplinas o saberes cercanos (Sociología, Antropología, Historia, en el ámbito de las Ciencias Sociales, o como Biología, Medicina y Química, en el de las Ciencias Naturales, entre otros), disciplinas o saberes afines (Sociología y Economía, por ejemplo), disciplinas o saberes distantes (Astrofísica y Antropología, por ejemplo), y disciplinas o saberes remotos (artes plásticas y matemáticas, entre otros). Así, si tomamos como punto central de referencia una disciplina y un saber cualquiera y lo situamos en un diagrama concéntrico, podemos clasificar todas las otras disciplinas y todos los otros saberes dentro de un esquema que exprese las distancias epistemológicas entre estas, de acuerdo con las categorías señaladas poco antes (cercanas, afines, distantes, remotas) (figura 2).

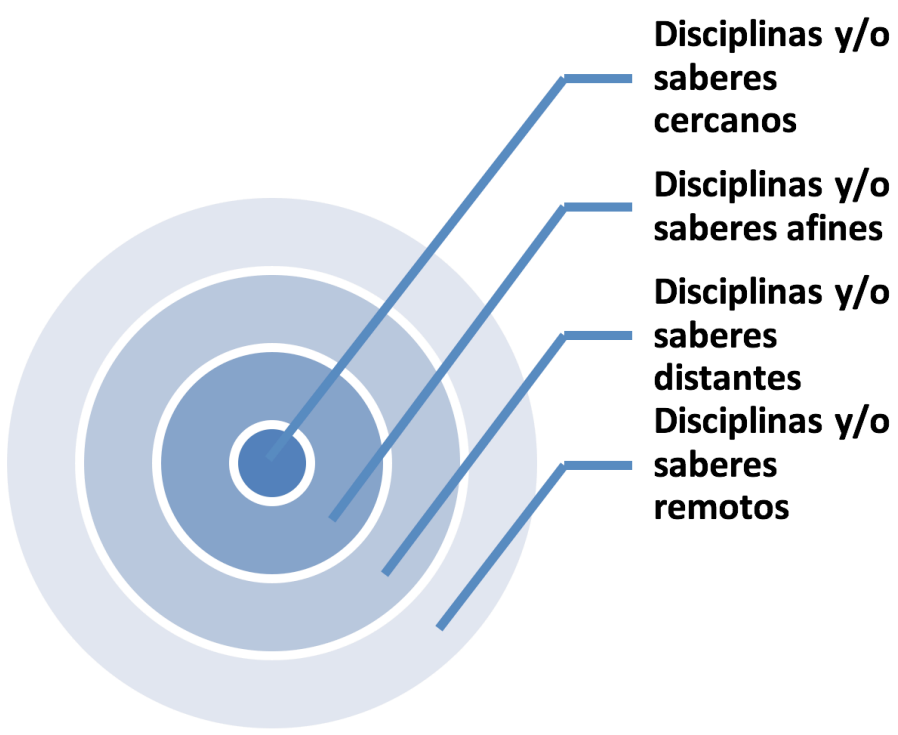

Figura 2. Distancias epistemológicas entre disciplinas y saberes 
En el CEG contamos con las tres categorías de disciplinas y para avanzar hacia la interdisciplina requerimos uno o varios paradigmas transversales (figura 1), que nos permitan establecer un marco conceptual y metodológico común para la integración interdisciplinaria de disciplinas de muy diferente naturaleza, dentro y fuera del campo de las diversas ciencias. Por ejemplo: ¿cómo integrar la Literatura, el Teatro y las Artes Plásticas con la Historia, la Biología, la Ecología y la Sociología? Esta es una de las grandes interrogantes que en este momento debemos resolver para transitar con éxito de la multidisciplina a la interdisciplina. Se trata, por consiguiente, de un doble desafío, ya que no es una mera integración interdisciplinaria entre las ciencias, sino de la fusión epistemológica de estas con disciplinas, saberes y modalidades de la praxis cognoscitiva humana, tan remotas como las artes.

Ante todo, es necesario aclarar y ponernos de acuerdo con los términos que empleamos y no por un prurito estrictamente semántico, sino porque la definición de ciertos vocablos claves será determinante en la vía epistemológica que decidamos proseguir para lograr la transición de la multidisciplina a la interdisciplina. Entendemos por multidisciplina cuando varias disciplinas trabajan dentro de un plan común de investigación y de trabajo, pero conservan sus fronteras disciplinarias específicas y con escasa integración cognoscitiva y metodológica entre ellas (figura 3) (Petrie 1976, 29-43 pp; Miller, Baird, Littlefield, Kofinas, Chapin \& Redman, 2008, 20-46 pp).

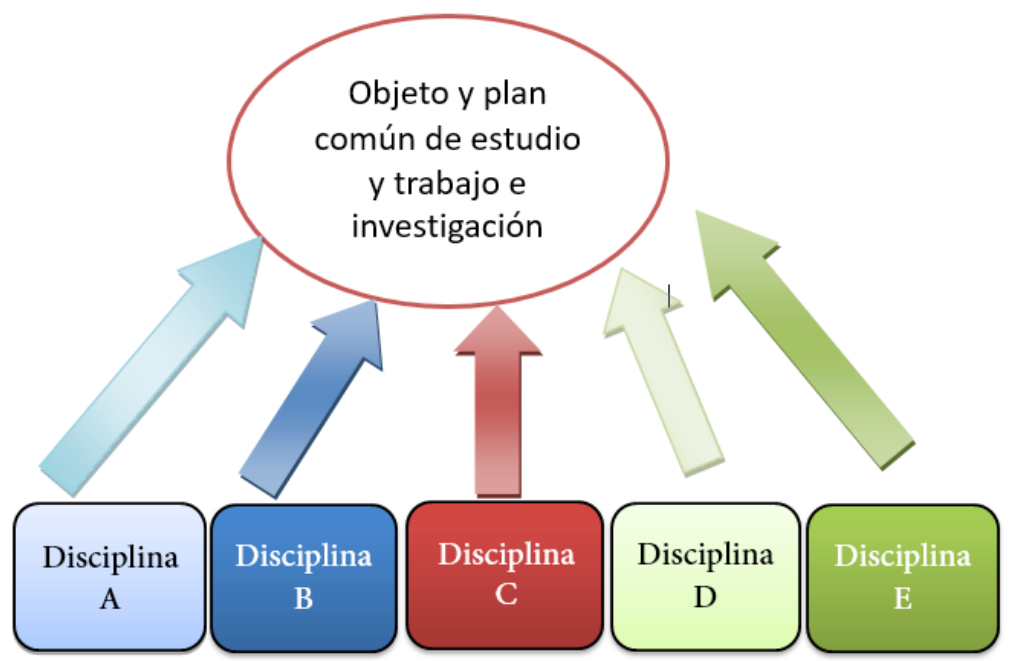

Figura 3. La multidisciplina 
Un ejemplo ilustrativo de una estrategia de investigación multidisciplinaria corresponde a los estudios de una determinada región geopolítica, donde diversos capítulos o secciones pertinentes la abordan y, por separado, desde ciertas temáticas particulares como: la geografía, los sistemas naturales, la demografía, la historia, la organización social y cultural, entre otros. En este tipo de investigación, cada disciplina aborda de forma específica e independiente los diversos temas que describen aspectos esenciales del objeto de estudio, pero sin establecer conexiones sistémicas y orgánicas e interdisciplinarias entre los diferentes aspectos y niveles fenomenológicos. El objeto de estudio multidisciplinario es así construido como un rompecabezas, formado por piezas separadas y autónomas, pero con el agravante de que el resultado que emerge no genera, por tanto, una visión integrada: son los diversos árboles reunidos, pero sin que ello permita visualizar el conjunto del bosque. Si esto se transfiere al terreno pedagógico, tenemos una docencia diversa, pero al mismo tiempo constituida por saberes muy fragmentados.

Frente a las limitaciones fragmentarias de la multidisciplina, la interdisciplina emerge como un nivel de comprensión más complejo, más concreto (donde el modelo de análisis y enseñanza reflejan más la riqueza concreta del mundo por conocer). Es cuando varias disciplinas se integran de manera muy estrecha dentro de un plan común de investigación, docencia, estudio o trabajo, y así las fronteras disciplinarias se disipan al amparo de metodologías y teorías transdisciplinarias (figuras 4 y 5 ).

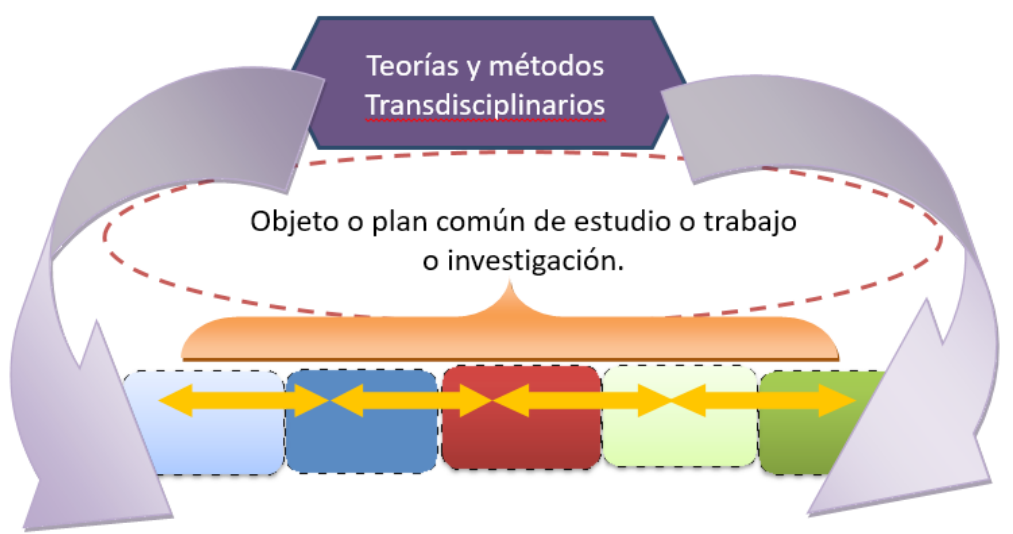

Figura 4. La interdisciplina que emerge de los paradigmas transdisciplinarios 
Por lo tanto, un paradigma transversal o transdisciplinario constituye una teoría (o conjunto de teorías) y una metodología (o conjunto de metodologías) que se ha nutrido de muchos conocimientos disciplinarios (figura 6), pero que los ha integrado en una visión holística que se encuentra por encima de las fronteras de las distintas disciplinas y que les brinda así un marco conceptual y metodológico que les permite a estas integrarse, a su vez, en una labor interdisciplinaria. La necesidad de la interdisciplina no surge como resultado de un esfuerzo epistemológico efectuado a-priori, sino como producto de modelos conceptuales y metodológicos que buscan explicar fenómenos de la realidad que solo pueden ser aprehendidos mediante el trabajo interdisciplinario.

Partimos del supuesto, entonces, de que la complejidad es una propiedad intrínseca de la realidad y que lo que varía es la mayor o menor complejidad-simplicidad de los modelos de análisis elaborados y empleados para entender esa realidad. Los modelos simples permiten así analizar aspectos simplificados de una realidad compleja, mientras que los modelos más complejos nos habilitan para entender la realidad desde una óptica más compleja, más concreta y más dialéctica.

Cuanto más interdisciplinarios sean los modelos de análisis, mayor es la riqueza intrínsecamente compleja de la realidad que podremos captar mediante teorías y métodos que solo la interdisciplina puede brindar. Es lógico, por consiguiente, postular que no es que la realidad sea simple o compleja, sino que son nuestros modelos de análisis los que la abordan de manera más simple o más compleja, sin poder nunca captarla en toda su verdadera complejidad total. 


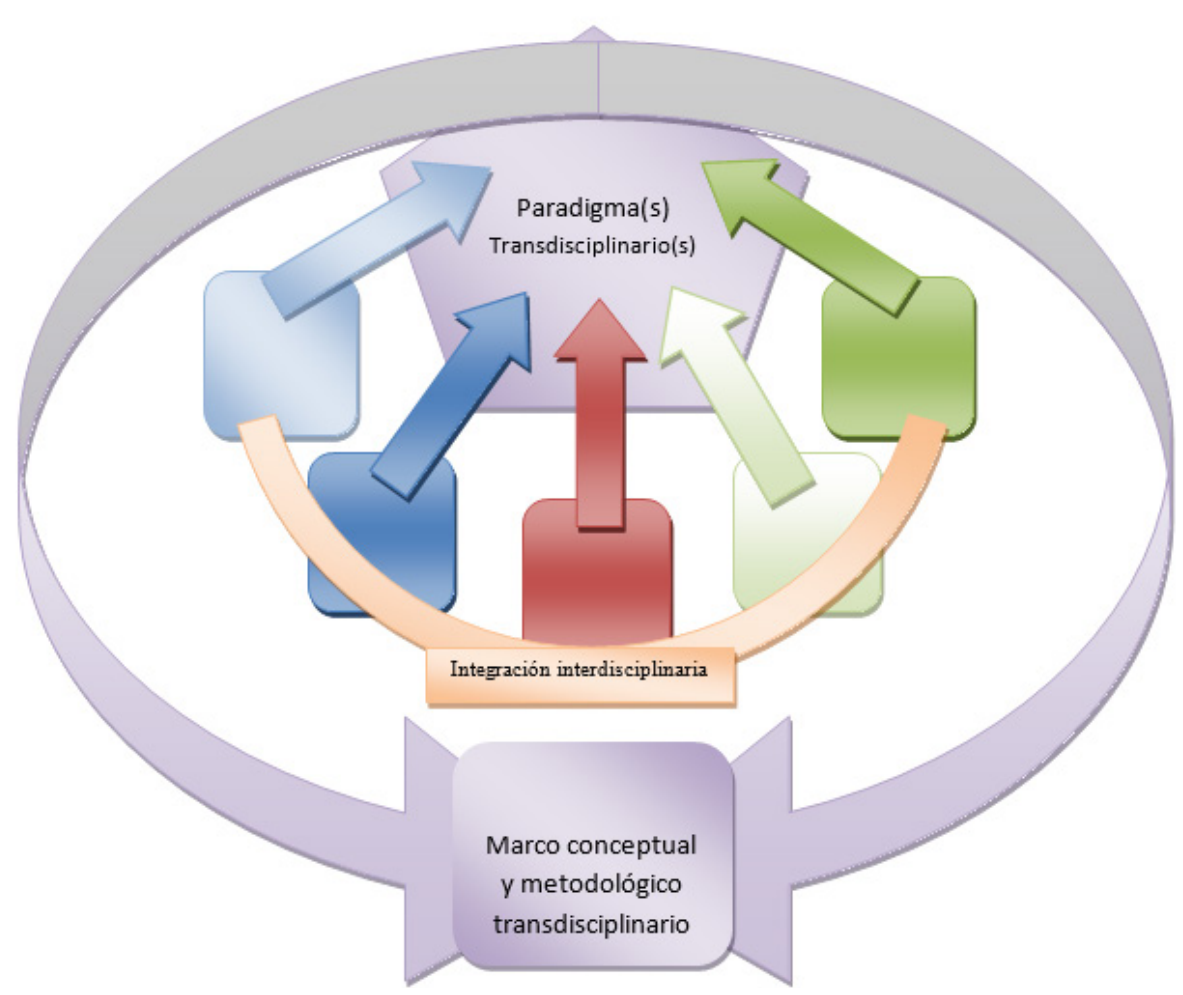

Figura 5. La interdisciplina nutre la transdisciplina y viceversa

Es obvio que los modelos interdisciplinarios de análisis representan un salto cualitativo en relación con los estrictamente disciplinarios o multidisciplinarios. La interdisciplina nos permite así elaborar modelos más complejos que, a su vez, nos dan mejor acceso a la complejidad intrínseca de la realidad, en un proceso cognoscitivo que es como una espiral que se amplía y asciende cada vez más hacia la concreción, la complejidad y la dialéctica, sin cesar y sin alcanzar nunca el giro final (Piaget y García 1983) A su vez, la interdisciplina solo puede conjugar las perspectivas epistemológicas variadas del conocimiento y de la praxis humana, mediante paradigmas transversales, es decir, con base en la transdisciplina. 


\section{Tabla 1}

Algunos ejemplos de formulaciones transdisciplinarias y su alcance epistemológico

\begin{tabular}{ll}
\hline \multicolumn{1}{c}{ Paradigma transversal } & \multicolumn{1}{c}{ Espectro disciplinario } \\
Estructuralismo & Conjunto de las ciencias sociales. \\
Funcionalismo & Economía, sociología e historia. \\
Materialismo histórico y dialéctico. & Para todas las ciencias. \\
Autopoyesis & Para todas las ciencias de la vida. \\
Teoría de sistemas & Para todas las ciencias. \\
Teoría del pensamiento complejo & Para todas las ciencias. \\
Ecología social & Para todas las ciencias de la vida. \\
Teoría de cuerdas & Para todos los saberes humanos \\
Nuevo humanismo & Para todos los saberes humanos. \\
\hline
\end{tabular}

Siguiendo esta perspectiva, las nuevas autoridades del CEG, Roberto Rojas Benavides, Decano y Miguel Baraona Cockerell, Vicedecano (a partir del segundo ciclo del 2015), desarrollaron un plan de acción para llevar a cabo esta transición de la multidisciplina a la interdisciplina, mediante el desarrollo y la aplicación del paradigma transversal del Nuevo Humanismo que se ha venido desarrollando en la unidad académica desde hace algunos años y que debe servir de marco transdisciplinario que permita comenzar este proceso en los próximos cinco años.

Ergo, tres principios epistemológicos inescapables para la interdisciplina:

1. Se necesita un paradigma transversal o transdisciplinario para llevarla a cabo con efectividad.

2. Mientras mayor sea la distancia epistemológica de las disciplinas y los saberes involucrados, más amplio debe ser también el carácter del paradigma transdisciplinario en cuestión.

3. Cuando se trata de un trabajo interdisciplinario que involucra saberes muy remotos, entonces no puede ser solo un paradigma de carácter científico, sino también de carácter ético-filosófico e incluso estético.

Ahora bien, ¿qué clase de teoría transdisciplinaria o paradigma transversal podemos desarrollar e implementar nosotros? 
Es un gran desafío, ya que se trata de elaborar un paradigma transversal que sea no solo aplicable a la interdisciplina entre disciplinas afines, o al menos cercanas, sino entre saberes tan remotos entre sí como las ciencias naturales y el arte. Y es aquí donde entra el Nuevo Humanismo, propuesta intelectual que podemos elevar a la categoría de un paradigma transdisciplinario de carácter ético-filosófico, de manera que permita la interdisciplina entre disciplinas y saberes cercanos, afines, distantes y remotos.

\section{Del Humanismo al Nuevo Humanismo}

El CEG-UNA se ha propuesto, desde sus orígenes, hace ya más de cuarenta años, adscribirse a una reflexión permanente sobre el humanismo, al buscar, además, aplicar sus diversos preceptos al conjunto de la vida académica e institucional del conjunto de la unidad. En el presente nos encontramos dedicados con intensidad a definir un paradigma transdisciplinario que nos ayude a alcanzar la integración interdisciplinaria y al cual hemos denominado Nuevo Humanismo.

Concebimos el Nuevo Humanismo como una teoría crítica y, al mismo tiempo, esperanzadora, sobre la condición humana en los albores del siglo XXI. En tanto teoría crítica, el Nuevo Humanismo se distingue por oponerse a:

a. Todo resabio colonial del pasado.

b. Toda forma de neocolonialismo actual, incluidas las modalidades más actuales de control mental de toda la población del orbe mediante el consumismo, el tecnologismo a ultranza, y el mercantilismo.

c. Toda forma de sexismo.

d. Toda forma de clasismo.

e. Toda forma de racismo.

f. Toda forma de alienación colectiva como: el consumismo, el tecnologísmo a ultranza y el mercantilismo. 
El Nuevo Humanismo propone:

- Una sociedad global libre de toda desigualdad, asimetría y discriminación internacional.

- Igualdad de género en derechos y oportunidades.

- Reducción de la desigualdad social.

- Una sociedad sin prejuicios ni discriminación étnica y racial, y sin xenofobia.

- Una sociedad en la que la libertad y la integridad moral y física del ser humano no esté supeditada al materialismo, a la tecnología y al lucro.

El Nuevo Humanismo abraza toda aquella doctrina que proponga el perfeccionamiento de la vida colectiva e individual humana, a partir de la confluencia creativa de la solidaridad como principio fundamental de interacción social, del pensamiento complejo como la mejor forma de entender el mundo y del biocentrismo, que sitúa al ser humano dentro de la totalidad de la naturaleza y no fuera de ella. Esta reformulación del humanismo la pensamos a través de lo que hemos llamado el paradigma tridimensional (figura 6).

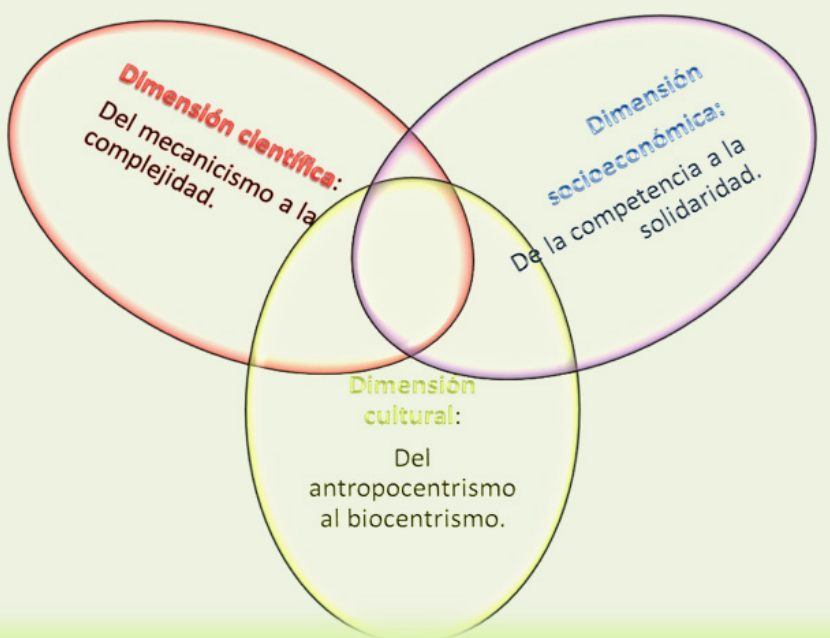

Figura 6: El Paradigma Tridimensional del Nuevo Humanismo 
A grandes rasgos, este paradigma tridimensional constituye una perspectiva ético-filosófica-científica, cuya naturaleza abordaremos con mayor profundidad y amplitud en un próximo trabajo. 3 Es necesario destacar, además, que se trata de una elaboración en pleno proceso. Los resultados próximos de este esfuerzo podrán seguirse a través de la Revista del Nuevo Humanismo del CEG-UNA y del próximo Boletín del Nuevo Humanismo, en el cual se intentará ofrecer un balance sintético y periódico de este trabajo colectivo del CEG.

\section{Balance y perspectivas}

Durante este quinquenio (2015-2020), estaremos avanzando en la dirección que, de manera tan esquemática, hemos presentado en este trabajo y estamos muy esperanzados de que la transición de la multidisciplina a la interdisciplina, como práctica académica del CEG en todas sus áreas sustantivas (docencia, investigación, extensión y producción) se materialice durante el ciclo de la actual decanatura.

El 16 y 17 de julio del 2015, el CEG se reunió en forma amplia y durante dos jornadas intensivas de trabajo se elaboró el documento "Pacto humanista del CEG", donde se establecen los pasos futuros por seguir para impulsar lo interdisciplinario dentro de esta unidad académica. En futuros trabajos seguiremos reseñando la experiencia, los altos y bajos y los frutos de este esfuerzo mancomunado que recién comienza.

Empleamos aquí el concepto de epistemología en su acepción más común, en tanto "teoría del conocimiento" (Bachelard, 1973).

Estamos conscientes de que cada una de estas tres nociones (consumismo, tecnologísmo a ultranza y mercantilismo) requiere de una elaboración más profunda, la que ya en parte hemos hecho en tres trabajos anteriores (Baraona, 2005, 2011 y 2013) y cuya complejidad excede el marco limitado de este artículo.

\section{Referencias}

Bachelard, G. (1973). Epistemología. Barcelona: Anagrama.

Baraona, M. (2005). Puntos de fuga: el fin de una historia y la transición a la hipermodernidad. Santiago de Chile: Editorial LOM.

Baraona, M. (2011). Humanismo para la hipermodernidad y sus alternativas. En Diez ensayos críticos. San José, Costa Rica. 
Baraona, M. (2013). Para un humanismo del siglo XXI: desafios y propuestas. En Revista de Nuevo Humanismo 1(1):11-21.

Davies, M. \& Marcia, D. (2007). Interdisciplinary Higher Education: Implications for Teaching and Learning. Australia-Melbourne: The Center for the Study of Higher Education.

Fonseca, Z. (2012). Historia del Centro Estudios General de la UNA. San José, Costa Rica. Documento de circulación interna. sp.

García, R. (2006). Sistemas complejos. Conceptos, método y fundamentación epistemológica de la investigación interdisciplinaria. Barcelona: Gedisa.

Kosic, K. (1967). Dialéctica de lo concreto. México: Editorial Grijalba.

Miller, TR, Baird, TD, C. M. Littlefield, CM, Kofinas, G, Chapin, F, \& Redman, CL (2008). Epistemological Pluralism: Reorganizing Interdisciplinary Research. Ecology and Society 13(2):20-46.

Petrie, G. (1976). Do You See What I See? The Epistemology of Interdisciplinary Inquiry. En Journal of Aesthetic Education 10(1): 29-43. University of Illinois Press. Recuperado de http://www.jstor.org/stable/3332007

Piaget, J y Rolando, G. (1983). Psychogenèse et Histoire des Sciences. France: Nouvelle Bibliotheque Scientifique-Flammarion.

Rojas, R y Baraona, M. (2015). Programa para el porvenir del CEG de la UNA. San José, Costa Rica. 